\title{
Evaluation of ovarian response prediction according to age and serum AMH levels in IVF cycles: a retrospective analysis
}

\author{
Sathy M. Pillai ${ }^{1}$, Nishtha A. Mahabalshetti ${ }^{2 *}$
}

\author{
${ }^{1}$ Samad IVF Hospital, Trivandrum, Kerala, India \\ ${ }^{2}$ Department of Obstetrics and Gynecology, SDM Medical College and Hospital, Dharwad, Karnataka, India
}

Received: 23 June 2017

Accepted: 07 July 2017

\section{*Correspondence:}

Dr. Nishtha A. Mahabalshetti,

E-mail: drnishtha.shetti@gmail.com

Copyright: (C) the author(s), publisher and licensee Medip Academy. This is an open-access article distributed under the terms of the Creative Commons Attribution Non-Commercial License, which permits unrestricted non-commercial use, distribution, and reproduction in any medium, provided the original work is properly cited.

\section{ABSTRACT}

Background: Increasing female literacy and employment have resulted in a clear rise in the age at which women conceive. As fertility starts to decline with advancing age more and more number of women are facing the problem of infertility and are seeking medical attention to overcome this problem. A number of ovarian reserve tests were developed to overcome this problem. Recent studies indicate that anti mullerian hormone is a promising marker for predicting ovarian reserve and pregnancy outcome.

Methods: A retrospective study conducted at SAMAD IVF hospital, Trivandrum, Kerala. The aim of this study was to assess the influence of age on Anti-Mullerian Hormone (AMH) in ART (assisted reproductive technology) program outcomes and determine whether AMH levels and age predict the availability of total oocytes and MII oocytes retrieved and good quality of embryos. Ninety-six subjects in the age group of 25 to 40 years were included and underwent controlled ovarian stimulation (COS) and later intracytoplasmic sperm injection (ICSI) was performed.

Results: In present study, it is seen that age and AMH (anti-mullerian hormone) have negative correlation. In accordance to AMH, total number of oocytes retrieved, MII oocytes, total number of embryos and grade A embryos were higher in AMH group ( $>5 \mathrm{ng} / \mathrm{ml}$ ). Total embryos for cryopreservation were lower in age group of 31-35yrs and $36-40 y$ rs with least AMH value as compared to younger age group, good quality of embryos was higher in age group of 25-30yrs with 1.5-5 and >5 AMH group.

Conclusions: Serum AMH is an important ovarian reserve test to predict response in ART cycles. Age is an independent marker in terms of quality of oocytes retrieved. We conclude that age and AMH have negative correlation. Hence, AMH can predict number of oocytes retrieved but age predicts quality of oocytes and embryos.

Keywords: Age, AMH, ART, Oocytes

\section{INTRODUCTION}

The accurate prediction of the response to ovarian stimulation is a valuable diagnostic step in the process of assisted reproduction. Increasing female literacy and employment have resulted in a clear rise in the age at which women conceive. As fertility starts to decline with advancing age more and more number of women are facing the problem of infertility and are seeking medical attention to overcome this problem. This decline in fertility is predominantly due to reduced ovarian reserve with increasing age. It has been shown that the rate of ovarian reserve decline varies considerably between individual women making it a challenge to design tests that estimate an individual's reproductive lifespan at a given age. ${ }^{1}$ A reliable marker for the age at which subfertility will occur would have great potential value as predictor of future reproductive lifespan. The ideal 
marker would show a significant change in levels from adolescence to late reproductive period. ${ }^{2}$ The primary value of ovarian reserve markers is to provide assistance in selecting an appropriate protocol and/or initial dose of gonadotrophin for controlled ovarian stimulation (COS) in IVF cycles. Some studies indicate that some of the biomarkers used for assessing ovarian reserve can also be used as predictors of pregnancy outcome in assisted reproduction programs. ${ }^{3,4}$ Recent studies indicate that Antimullerian hormone (AMH), a glycoprotein can be used as a promising marker for predicting ovarian reserve and pregnancyoutcome..$^{5-7}$

Anti mullerianhormone (AMH) is a dimeric glycoprotein belonging to the transforming growth factor (TGF- $\beta$ ) super family, which acts on tissue growth and differentiation. In the female, it is a product of the granulosa cells from preantral and small antral follicles. ${ }^{8}$ In females, AMH appears to have inhibitory effect upon the recruitment of primordial follicles and it may decrease the sensitivity of large pre-antral and small antral follicles to FSH. AMH expression declines as antral follicles increase in size with nominal expression restricted to the granulosa cells of the cumulus. This loss of $\mathrm{AMH}$ expression during the $\mathrm{FSH}$ dependent final stages of follicular growth, and the lack of expression by atretic follicles suggest that basal levels of AMH may more accurately reflect the total developing follicular cohort and consequently potential ovarian response to FSH.

Furthermore, AMH has been shown not to fluctuate across the menstrual cycle consistent with its role reflecting the continuous non-cyclic growth of small follicles in the ovary. ${ }^{3}$ In harmony with the established relationship between age and declining ovarian reserve, AMH falls linearly with increasing age. This occurs in conjuction with reductions in the antral follicular count, which is strongly correlated, to plasma AMH levels. $\mathrm{AMH}$ has consequently been explored as a predictor of ovarian response to FSH and oocyte quality in cycles of ART. ${ }^{9}$ More over AMH levels have better cycle-to-cycle reproducibility. Some recent studies have shown AMH as predictor of pregnancy outcome in assisted reproductive techniques. $^{10,11}$

\section{METHODS}

This is a retrospective study conducted from Janauary 2015 to June 2016 at Samad IVF Hospital, Trivandrum. A total of 96 patients were chosen, who were in their first IVF cycle, age <40 years, with regular menstrual cycles, euthyroid and normal prolactin levels, basal serum FSH $<12 \mathrm{mIU} / \mathrm{ml}$ and BMI of $<30 \mathrm{~kg} / \mathrm{m}^{2}$, no male factor cause for infertility were included. Patients were classified according to $\mathrm{AMH}$ levels as

- $\quad$ Group I (0.5-1.5ng/ml)

- $\quad$ Group II (1.6-5ng/ml)

- Group III (more than 5ng/ml)
Patients were classified according to age

- $\quad$ Between 25-30 years

- 31-35 years

- 36-40 years.

The initial survey for the etiology of infertility included a routine gynecological examination, a basic vaginal ultrasound, saline sonography or Laparoscopy for evaluation of tubal factors, HbA1c and semen analysis of the male partner. Baseline hormone profiles including serum levels of estradiol, progesterone, FSH (follicle stimulating hormone), LH (luteinizing hormone) and $\mathrm{AMH}$ were determined on day three of the previous cycle.

All patients received antagonist (flexible) protocol. The ovarian response was monitored by TVS and serum E2 levels, the dose of gonadotropins adjusted accordingly. When 3 or more follicles reached a maximum diameter of $18 \mathrm{~mm}$, rHCG 250microgram was given as trigger, blood drawn for E2 measurement, oocyte retreival done after 34-36hrs of trigger, ICSI was performed. Embryos were cryopreserved and patients were planned for frozen embryo transfer.

Serum sample for peak E2 was collected on the day of HCG. Patients were studied with respect to total number of oocytes, number of mature oocytes, duration of stimulation, total dose of gonadotropins, peak E2 levels, number of embryos and Grade A embryos. According to number of oocytes retreived, patients were divided into poor responders (<3 oocytes) normal responders (4-19) and hyperresponders $(>20)$.

\section{Statistical analysis}

Data was statistically analysed. One Way Anova test, chi square test and Pearson's correlation was calculated.

\section{RESULTS}

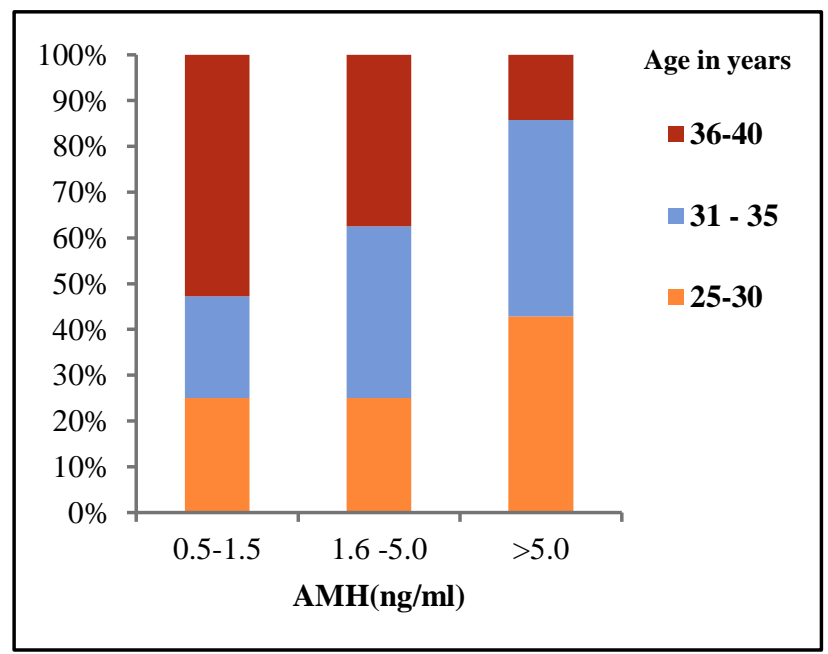

Figure 1: Correlation between age in years and AMH. 
Among 96 patients, 30.2\% were between 25-30 years, $33.3 \%$ were between $31-35$ years and $36.5 \%$ were between 35-40 years. According to AMH levels, Group I $(0.5-1.5 \mathrm{ng} / \mathrm{ml})$ were $37.5 \%$ patients, Group II (1.6$5 \mathrm{ng} / \mathrm{ml})$ were $33.3 \%$ and Group III $(>5 \mathrm{ng} / \mathrm{ml})$ were $29.2 \%$ patients. In the present study, correlation between age and AMH was calculated, and was found that there was negative correlation, indicating that as age advances AMH levels decline as in Figure 1. All patients $(n=96)$ were grouped according to their age (Table 1). Parameters in relation to controlled ovarian stimulation were assessed which were BMI $\left(\mathrm{kg} / \mathrm{m}^{2}\right)$, total number of days required for stimulation, total dose of gonadotropins used for stimulation (IU), serum peak estradiol (E2) levels $(\mathrm{pg} / \mathrm{ml})$, total oocytes retrieved, number of mature (MII) oocytes, total embryos and good quality of embryos according to their morphology (Grade A). BMI, number of days for stimulation and total dose of gonadotropins required throughout stimulation had no statistical significance, though number of days and total dose increased as age advances (highest in 36-40 yrs group).

Table 1: Group characteristics according to age.

\begin{tabular}{|c|c|c|c|c|c|c|c|}
\hline & Age in : & & & & & & \\
\hline & $25-30(1$ & & 31-35 (l & & $36-40($ & & \\
\hline & Mean & SD & Mean & SD & Mean & SD & $\mathbf{p}$ \\
\hline BMI & 25.9 & 2.9 & 26.4 & 2.4 & 26.3 & 2.1 & 0.687 \\
\hline No of days & 9.6 & 2.4 & 10.7 & 4.4 & 10.1 & 2.9 & 0.446 \\
\hline Total dose(IU) & 2136.2 & 834.1 & 2274.8 & 661.1 & 2552.1 & 866.4 & 0.105 \\
\hline E2 levels (pg/ml) & 1537.9 & 776.0 & 2012.1 & 1227.4 & 1294.2 & 839.1 & $<0.002$ \\
\hline Number of total oocytes & 8.41 & 4.26 & 8.45 & 5.47 & 6.46 & 4.09 & 0.139 \\
\hline Number of mature oocytes & 4.31 & 2.45 & 4.53 & 2.95 & 3.63 & 2.39 & $<0.001$ \\
\hline Total number of embryos & 4.62 & 2.56 & 4.59 & 3.43 & 3.43 & 2.42 & 0.152 \\
\hline Grade A embryos & 0.97 & 1.09 & 1.22 & 1.18 & 0.86 & 1.00 & 0.389 \\
\hline
\end{tabular}

Patients were grouped according to their age. Data shown are mean+ SD, Chi square test and One-way Anova test were used to analyse. Parameters in relation to controlled ovarian stimulation were assessed. Significant $\mathrm{p}$ value $(0.005)$ was seen in relation to peak serum estradiol (E2) levels and number of mature oocytes among different age groups (Table 1).

Table 2: Group characteristics according to AMH levels.

\begin{tabular}{|c|c|c|c|c|c|c|c|}
\hline & \multicolumn{7}{|l|}{ AMH } \\
\hline & \multicolumn{2}{|c|}{$0.5-1.5(\mathrm{~N}=36)$} & \multicolumn{2}{|c|}{$1.6-5.0(\mathrm{~N}=32)$} & \multicolumn{2}{|c|}{$>5.0(\mathrm{~N}=28)$} & \multirow{2}{*}{$\mathbf{p}$} \\
\hline & mean & sd & mean & sd & mean & sd & \\
\hline BMI & 26.3 & 2.3 & 24.84 & 1.91 & 27.78 & 2.41 & $<0.001$ \\
\hline No of days & 11.3 & 2.9 & 9.47 & 3.15 & 9.50 & 3.78 & 0.031 \\
\hline Total dose & 3022.2 & 676.6 & 2020.31 & 576.99 & 1807.86 & 515.20 & $<0.001$ \\
\hline E2 levels & 1072.4 & 586.4 & 1735.84 & 1034.49 & 2147.57 & 1090.39 & $<0.001$ \\
\hline Number of total oocytes & 4.47 & 2.36 & 8.48 & 3.89 & 11.00 & 5.19 & $<0.001$ \\
\hline Number of Mature oocytes & 2.44 & 1.32 & 4.28 & 2.11 & 6.14 & 2.93 & $<0.001$ \\
\hline Total number of embryos & 2.67 & 1.94 & 4.28 & 2.28 & 6.00 & 3.39 & $<0.001$ \\
\hline Grade A Embryos & 0.67 & 0.83 & 1.06 & 1.13 & 1.39 & 1.23 & 0.027 \\
\hline
\end{tabular}

The total dose of gonadotropins required was more among the AMH group $(0.5-1.5 \mathrm{ng} / \mathrm{ml})$, peak serum E2 levels on day of HCG trigger was seen to be least with low AMH group, total number of oocytes retrieved, number of mature oocytes and total number of embryos and Grade A embryos were less in low AMH group (0.5$1.5 \mathrm{ng} / \mathrm{ml})$ compared to other AMH group, which was statistically significant. Patients were grouped according to $\mathrm{AMH}$ levels(ng/ml).

Data shown as mean+SD values, Chi square test and One-way Anova test were used to analyse. Parameters in relation to controlled ovarian stimulation were assessed as seen in Table 2. 
According to the number of oocytes retrieved, the patients were classified as poor responders ( $<3$ oocytes), normo responders (4-19 oocytes) and hyper responders (>20 oocytes) in both AMH group and Age group. Among AMH, Group I (0.5-1.5ng/ml) 55.6\% were poor responders, $44.4 \%$ were normo responders and none were hyper responders, in Group II (1.6-5ng/ml) $18.8 \%$ were poor resonders, $81.3 \%$ were normo responders, and in Group III $(>5 \mathrm{ng} / \mathrm{ml}) 7.1 \%, 89.3 \%$ and $3.6 \%$ were poor, normo and hyper responders respectively. $\mathrm{P}$ value was statistically significant $(\mathrm{p}<0.001)$. According to age, Group I (25-30yrs) $24.1 \%, 75.9 \%$ were poor and normo responders. Group II (31-35yrs) $21.9 \%, 75 \%$ and $3.1 \%$ were poor, normo and hyper responders whereas in Group III (36-40yrs) $40 \%$ and $60 \%$ were poor and normo responders respectively. $P$ value was calculated $(p=0.287)$ as in Figure 2.

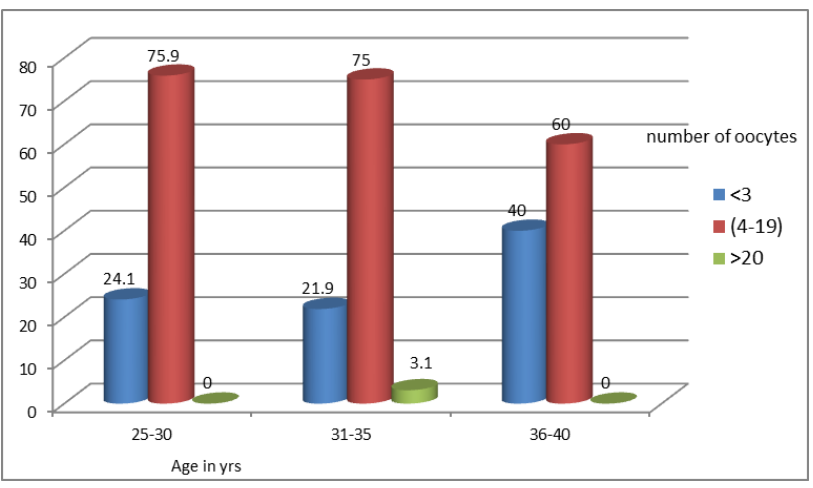

Figure 2: Comparison of total number of oocytes with age.

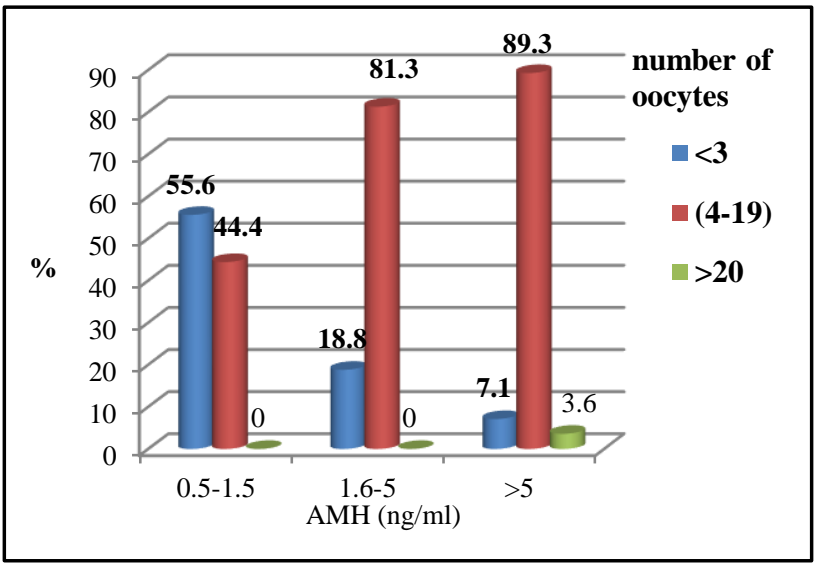

Figure 3: Comparison of total number of oocytes with Age and AMH.

\section{DISCUSSION}

The success of assisted reproduction technologies is jointly determined by the interaction between oocyte quality and quantity. The ovarian ageing process results qualitatively from the decline in the proportion of developmentally competent oocytes as well as quantitatively from the diminishing number of oocytes available for selection and recruitment. However, with ovarian ageing, the diminishing proportion of normal oocytes needs to be compensated for quantitatively by increasing the number of available oocytes through controlled ovarian hyperstimulation.

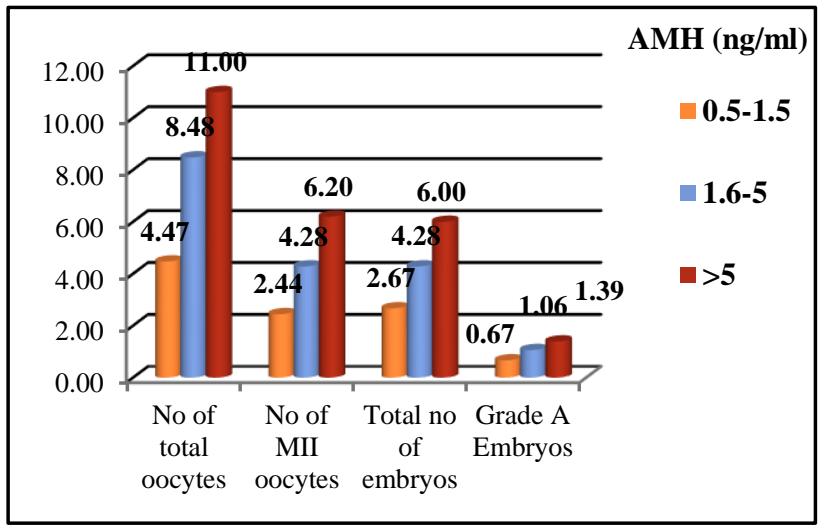

Figure 4: AMH with Number of oocytes, MII oocyte, total embryos and grade A embryo.

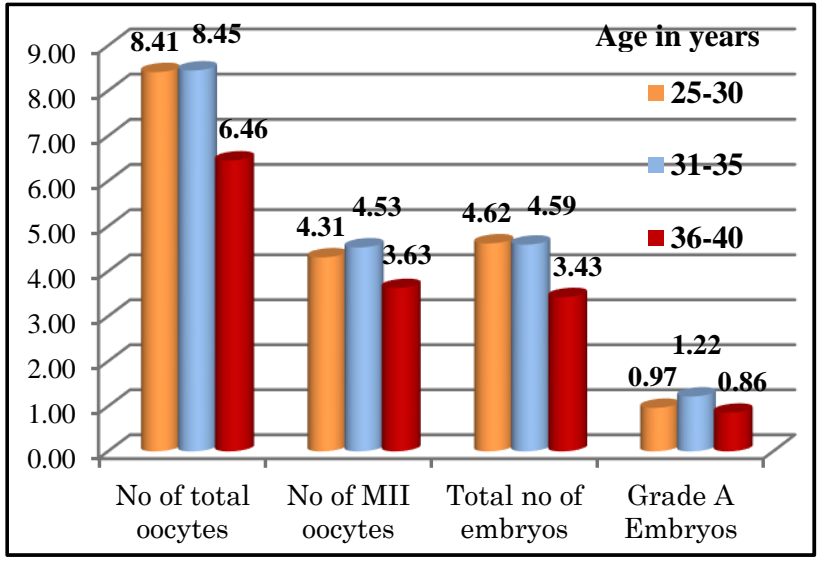

Figure 5: Age with Number of oocytes, MII oocyte, total embryos and grade $A$ embryos

This study shows inverse correlation between age and AMH (Figure 1). These observations are in accordance with documented evidence that fertility decreases with increasing age and AMH levels fall linearly with increasing age. ${ }^{1,9}$ Statistically significant differences were seen in the ovarian reserve parameters among the groups studied. Ovarian reserve parameters like AMH, no of retrieved oocytes, mature oocytes (MII) and grade A embryos were relatively higher in the age groups below 31-35 years.

Positive correlations were found between AMH levels and number of retrieved oocytes but not with other ovarian reserve parameters in the age group below 35 years. In the above 35 years age group, there were positive correlations between AMH levels and all other ovarian reserve parameters. In another recent study, the authors have reported positive predictive value for AMH in ART in women above 35 years of age and they also 
correlated pregnancy outcome to the etiology of infertility. ${ }^{10,11}$ From the present study, we conclude that age and $\mathrm{AMH}$ have negative correlation (r-0.266) (Figure $1)$.

In accordance to $\mathrm{AMH}$, total number of oocytes retrieved, mature oocytes, total number of embryos and good quality embryos were higher in AMH group (>5ng/ml). Total number of days required for stimulation was significantly higher in age group of 36-40yrs with AMH $(0.5-1.5 \mathrm{ng} / \mathrm{ml})$. Total oocytes and mature oocytes were higher in 25-30yrs age with AMH $(>5 \mathrm{ng} / \mathrm{ml})$ Total embryos for cryopreservation were lower in age group of 31-35 yrs and 36-40 yrs with least AMH value as compared to younger age group, also good quality of embryos was higher in age group of 25-30 yrs with 1.5-5 and $>5 \mathrm{ng} / \mathrm{ml}$ AMH group. Therefore, in present study, it was concluded that age gives better quality of oocytes, $\mathrm{AMH}$ predicts number of oocytes retrieved and age predicts quality of oocytes. At the present time one of the principal impediments to the more widespread use of $\mathrm{AMH}$ to predict IVF outcome is the lack of standardization of AMH assay results. Standardization of AMH assay results and more number of studies with larger sample size are required to establish $\mathrm{AMH}$ as a predictor of pregnancy outcome in this age group.

\section{CONCLUSION}

AMH seems to be a reliable and better ovarian reserve marker compared to age for prediction of IVF outcome in ART cycles, thus enabling reproductive clinicians to practice single embryo transfer with confidence and reduce the complications associated with multiple embryo transfer and need for fetal reduction. A simple age stratified analysis demonstrated variable relationship between quantitative ovarian reserve and oocyte quality, hence clinical application of basal AMH testing as predictor of IVF outcome may be appropriate for elderly aged women.

Funding: No funding sources

Conflict of interest: None declared

Ethical approval: The study was approved by the Institutional Ethics Committee

\section{REFERENCES}

1. Broer SL, Mol BW, Hendriks D, Broekmans FJ. The role of Antimullerian hormone in prediction of outcome after IVF: comparison with the antral follicle count. Fertil Steril. 2009;91:705-14.
2. La Marca A, Broekmans FJ, Volpe A, Fauser BC, Macklon NS. Anti-Mullerian hormone (AMH): what do we still need to know? Hum Reprod. 2009;24:2264-75.

3. Cheng-H HW, Yu-CC, Hsin-HW, Jyuer GY, YuJun C, Horng-Der T. Serum Antimullerian Hormone Predicts ovarian response and cycle out come in IVF patients. J Assist Reprod Genet. 2009;26:383-9.

4. Firouzabadi RD, Tayebi N, Asgharnia M. Serum level of Antimullerian Hormone in early follicular phage as a predictor of ovarian reserve and pregnancy outcome. Assisted reproductive technology cycles. Archiv Iran Med. 2008;11:371-6.

5. Nelson SM, Yates RW, Lyall H, Jamieson M, Traynor I, Gaudoin M et al. Antimullerian Hormone based approach to controlled ovarian stimulation for assisted conception. Hum reprod. 2009;24:867-75.

6. Lekamge DN, Barry M, Kolo M, Lane M, Gilchrist $\mathrm{RB}$, Tremellen KP. Anti-Mullerian hormone as a predictor of IVF outcome. Reprod Biomed. 2007;14:602-10.

7. Sriharibabu M, Himabindu Y, Gopinathan KK. Association of day 3 antimullerian hormone (AMH) with oocyte quality, fertilization rates and grade 1 embryos. Biomedicine. 2012;36:61-66.

8. Ebner T, Sommer MG, Moser MO, Shebl ES, Lechnerand GT. Basal level of Antimullerian Hormone is associated with oocytes quality in stimulated cycles. Human Reprod. 2006;21:2022-6.

9. Majumder K, Gelbaya TA, Laing I, Nard LG. The use of anti-mullerian hormone and antral follicle count to predict the potential of oocytes and embryos. Eur J Obstet Gynecol Reprod Biol. 2010;150:166-170.

10. Aflatoonian A, Oskouian H, Ahmadi S, Oskouian L. Prediction of high ovarian response to controlled ovarian hyperstimulation: antimullerian hormone versus small antral follicle count (2- $6 \mathrm{~mm})$. J Assist Repro Genet. 2009;26:319-321.

11. Lee TH, Liu CH, Huang CC, Hsieh KC, Lin PM, Lee MS. Impact of female age and male infertility on ovarian reserve markers to predict outcome of assisted reproduction technology cycles. Reprod Biol Endocrinol. 2009;7(1):100.

Cite this article as: Pillai SM, Mahabalshetti NA.

Evaluation of ovarian response prediction according to age and serum AMH levels in IVF cycles: a retrospective analysis. Int J Reprod Contracept Obstet Gynecol 2017;6:3306-10. 